Revue

Revue de l'histoire des religions

del'histoire des religions

\title{
Espace et structures cultuels du sanctuaire grec : la construction du vocabulaire
}

Cult Space and Structures of the Greek Sanctuary: Defining the Vocabulary

\author{
loanna Patera
}

\section{OpenEdition \\ Journals}

Édition électronique

URL : https://journals.openedition.org/rhr/7669

DOI : $10.4000 /$ rhr.7669

ISSN : 2105-2573

\section{Éditeur}

Armand Colin

\section{Édition imprimée}

Date de publication : 1 décembre 2010

Pagination : 535-551

ISBN : 978-2200-92658-8

ISSN : 0035-1423

\section{Référence électronique}

Ioanna Patera, «Espace et structures cultuels du sanctuaire grec : la construction du vocabulaire », Revue de l'histoire des religions [En ligne], 4| 2010, mis en ligne le 01 décembre 2013, consulté le 21 septembre 2021. URL : http://journals.openedition.org/rhr/7669; DOI : https://doi.org/10.4000/rhr 7669 


\section{Espace et structures cultuels du sanctuaire grec : la construction du vocabulaire}

Le vocabulaire généralement en usage pour décrire un sanctuaire et ses structures cultuelles implique un parti pris relatif à la fonction de ces structures. La terminologie la plus courante est examinée afin de clarifier ses champs sémantiques et ses emplois modernes. Cet examen mène à reconsidérer l'espace du sanctuaire, le hieron et le temenos, ainsi que ses limites. Certains termes désignant des composantes de l'espace sacré, notamment le naos et le thêsauros, sont également revus.

\section{Cult Space and Structures of the Greek Sanctuary : Defining the Vocabulary}

The vocabulary traditionally used to describe the area and the cultrelated structures of a sanctuary has led to a considerable confusion about their actual function. A closer examination of the current terminology will make it possible to clarify its semantic fields and its modern uses. I will reconsider the area of the sanctuary, the hieron and the temenos, and its boundaries. Finally, some terms designating components of sacred space, such as the naos and the thesaurus, will be also reconsidered. 
Pollux, le lexicographe du $\mathrm{II}^{\mathrm{e}}$ siècle de notre ère, fournit dans son Onomasticon une liste de termes techniques désignant les lieux de culte. Son commentaire montre l'ambivalence de la plupart d'entre eux :

«L'emplacement (chôrion) où se pratique le culte des dieux, c'est un hieron (sanctuaire) ou un neôs (temple), et là où nous procédons à une fondation (entha de kathidruomen) c'est un sêkos et un temenos [...]; les plus précis parlent de sêkos pour les héros, mais les poètes parlent aussi du sêkos des dieux [...]. Ce qui est devant lui c'est le prodomos, derrière, l'opisthodomos, l'entrée ce sont les propylées [...]. S'il y a un endroit du sanctuaire (hieron) où l'on ne peut marcher, un abaton, on peut aussi l'appeler aduton (lieu où l'on ne peut pénétrer), apsauston, apsaustoumenon (lieu auquel on ne peut toucher), atheaton (qu'on ne peut voir) et anaktoron (temple ou palais). Les lieux consacrés aux dieux (aneimenoi theois topoi) sont les alsê (bois sacrés), les temenê (enceintes) et les herkê (enclos), le cercle qui les entoure le peribolos ${ }^{1}$. »

Ces mêmes termes sont couramment repris par les modernes pour décrire les lieux de culte. Toutefois, en utilisant des termes antiques ambigus pour désigner des structures dont nous connaissons mal la fonction, on est enclin à des erreurs d'interprétation ${ }^{2}$.

Les problèmes méthodologiques liés à la terminologie des lieux de culte relèvent principalement de deux héritages intellectuels. Le premier est celui de l'école allemande de la science de l'antiquité du XIX ${ }^{\mathrm{e}}$ siècle qui prône le positivisme ${ }^{3}$. En même temps que la philologie se développe en science, avec des présupposés qui ne sont

1. Pollux, I, 6-11.

2. Olivier de Cazanove et John Scheid - "Aux sources d'un colloque», dans Olivier de Cazanove et John Scheid (dir.), Sanctuaires et sources dans l'antiquité. Les sources documentaires et leurs limites dans la description des lieux de culte. Actes de la table ronde organisée par le Collège de France, l'UMR 8585 Centre Gustave-Glotz, l'EFR et le Centre Jean Bérard, Naples, Centre Jean Bérard, 30 novembre 2001, Naples 2003, p. 1-6 (3) - qualifient cette habitude de recours à « un vocabulaire (pseudo-)antique, d'usage courant en archéologie, mais qui n'en demeure pas moins source de confusions ».

3. James Whitley, The Archaeology of Ancient Greece, Cambridge 2001, p. 32-33. Anthony M. Snodgrass, An Archaeology of Greece. The present state and future scope of a discipline, Berkeley-Los Angeles-Londres 1987, p. 37-38, dénonce de son côté les formes variées que revêt le positivisme dont souffre l'archéologie en tant que discipline, en particulier une forme de "positivist fallacy », consistant à associer ce qui est important au point de vue archéologique à ce qui est significatif au point de vue historique, l'observable au signifiant. 
ni ne doivent être questionnés, la rigueur devient une sorte d'idéal scientifique. Cette prétention s'est révélée plus tardivement dans la discipline de l'archéologie. Les fouilles d'Olympie (1875-1881), les premières à mettre en œuvre une technique stratigraphique rigoureuse, constituent une entreprise énorme autant en investissement humain et financier qu'en charge symbolique ${ }^{4}$. Mais les archéologues allemands sont avant tout des philologues attachés à l'apogée spirituel de la Grèce antique ${ }^{5}$. Par conséquent, la recherche de sites archéologiques s'accompagne de leur identification aux sites homériques, entraînant les erreurs que provoque la volonté d'illustrer la tradition littéraire au moyen de vestiges.

Le deuxième héritage qui nous induit à la généralisation et, souvent, à l'erreur, est celui de la terminologie antique elle-même, dont l'ambiguité est amplifiée par l'usage qu'en font les modernes. Vitruve déjà, au $\mathrm{I}^{\mathrm{er}}$ siècle avant notre ère, étudie l'architecture sur la base d'exemples grecs. Son troisième livre consacré aux temples, aux plans et aux proportions des sacrarum aedium, utilise la terminologie grecque qui reste encore en usage.

Lorsqu' on tente d'identifier un "espace sacré », juxtaposant ainsi deux concepts qu'il conviendrait d'examiner dans leur propre contexte d'énonciation ${ }^{6}$, nous recherchons certaines constantes qui caractérisent les lieux réservés aux dieux, relevant à la fois des structures et du mobilier. Les difficultés surgissent en même temps que nous tentons de les décrire en utilisant les termes techniques à notre disposition. Rencontrés dans différents types de sources, littéraires et épigraphiques, durant un laps de temps très long, ils ont dans ces sources antiques des sens variés et présentent des nuances multiples. Ce que nous décrivons est donc immédiatement interprété, en termes modernes, comme une structure ayant une fonction définie, désignée par un vocable auquel nous avons infligé une fixité qui ignore toute nuance et nous incite à lui attribuer la plus grande charge symbolique possible. Qui douterait par

4. Pour les fouilles d'Olympie et leur rapport à la politique, voir Lutz Klinkhammer, "Großgrabung und große Politik. Der Olympia-Vertrag als Epochenwende », dans Helmut Kyrieleis (éd.), Olympia 1875-2000. 125 Jahre Deutsche Ausgrabungen. Internationales Symposion, Berlin 9.-11. November 2000, Mainz am Rhein 2002, p. 31-47 (39-43).

5. J. Whitley, The Archaeology of Ancient Greece, p. 35.

6. Sur l'espace en tant que concept, voir David Clarke, «Archaeology: the Loss of Innocence », Antiquity 47 (1973), p. 6-18 (13). 
exemple que le « temple » Parthénon n'était en réalité pas un lieu de culte?

Un jalon historiographique important sur ces questions est celui de l'ouvrage désormais classique de Birgitta Bergquist concernant le temenos grec paru en 1967. Elle y déplore le fait que l'on nie aux sanctuaires grecs une structure fonctionnelle et qu'on les considère comme des ensembles désordonnés et accidentels ${ }^{7}$. Outre des questions de détail sur des sites particuliers, c'est son hypothèse des « éléments nécessaires » qui mérite d'être réexaminée. Les sanctuaires monumentaux possèdent en effet des éléments destinés à remplir des fonctions rituelles précises; ils semblent fréquents au point que Bergquist les considère comme caractéristiques des lieux de culte. S'il parait impossible de satisfaire à son ambition qui était de définir de quelle façon étaient arrangés les sanctuaires grecs et pourquoi ils étaient arrangés de cette façon ${ }^{8}$, nous pouvons tenter de définir des points plus restreints, moins ambitieux et néanmoins tout aussi problématiques.

L'élément fréquent, caractéristique, serait selon Bergquist le temenos, l'aire découpée, réservée à une divinité ou à un héros, avec le couple formé par le temple et l'autel. Il est dès lors nécessaire de revenir sur les terres sacrées et sur leurs délimitations.

\section{L'ESPACE CULTUEL ET SES LIMITES}

Dans l'Edipe à Colone de Sophocle, lorsqu'Edipe demande où il peut s'asseoir, en un endroit où l'on peut mettre le pied (bebêlois) ou dans les bois sacrés des dieux (alsesin theôn) ${ }^{9}$, Antigone suggère dans sa réponse que l'aspect de la nature suffit à prouver la sacralité des lieux : «ce lieu est sacré, cela est clair, il est couvert de lauriers, d'oliviers, de vignes et, là-dedans, sous ces feuillages, les rossignols chantent agréablement $\rangle^{10}$. Elle lui propose de se reposer sur une pierre. Mais l'étranger qui arrive lui dit de commencer

7. Birgitta Bergquist, The Archaic Greek Temenos. A Study of Structure and Function, Lund 1967, p. 1.

8. Ibid., p. 2.

9. Sophocle, Edipe à Colone, 9-10.

10. Ibid., 16-18. Pour les lieux naturels sacrés, voir Jean Rudhardt, « La perception grecque du territoire sacré », Mélanges de l'École française de Rome. Antiquité 113 (2001), p. 175-188 (178). 
par quitter ce siège, car il se trouve dans un endroit où il n'est pas pur de mettre le pied (ouch hagnon patein) ${ }^{11}$. Le chœur reconnaît en CEdipe un étranger, sans quoi jamais il ne serait entré dans le bois. Pour qu'il puisse parler, ils l'invitent à sortir de ce qui apparaît comme un périmètre interdit : " Change de place, sors de là [...] si tu as quelque chose à dire à notre groupe, sors du lieu où l'on ne peut marcher (abatôn apobas), et à l'endroit où chacun peut parler, parle $^{12}$. » Il ne doit pas dépasser le rocher qui se trouve là; il peut s’y asseoir « penché, de côté, au bout de la pierre ${ }^{13}$ ».

Bien que l'espace interdit de Colone ne soit pas marqué de limites visibles, celles-ci sont bien connues des habitants du pays. Ailleurs, les limites sont tangibles, matérialisées notamment par des bornes ${ }^{14}$. En 228-226 avant notre ère, « le sanctuaire d'Apollon Ptôios à Akraiphia, celui qui est délimité par des stèles (ai stêlai horizôsin), comme l'est aussi le sanctuaire de Delphes, est un asile; personne ne doit porter atteinte au reste de la terre sacrée (chôra hiera) d'Apollon Ptôios $\gg^{15}$. Cette hiera chôra peut consister en terrains réservés aux cultures ou aux troupeaux du sanctuaire ${ }^{16}$. Il en va ainsi à Delphes, bien que le règlement concernant ses pâturages puisse impliquer qu'une autre partie de la terre sacrée était accessible aux troupeaux des particuliers ${ }^{17}$. Les bornes elles-mêmes et la délimitation qu'elles marquent peuvent être contestées. C'est le propos du règlement athénien de 352/1 relatif à l'orgas sacrée

11. Sophocle, Edipe à Colone, 37.

12. Ibid., 162-169.

13. Ibid., 195-196.

14. Pour les bornes, voir Marietta Horster, Landbesitz griechischer Heiligtümer in archaischer und klassischer Zeit, Berlin-New York 2004, p. 25-29.

15. IG VII 4135, A 5-8 (= LSCG 73).

16. Sur les sens des termes chôros, chôrê et topos dans les sources littéraires, voir Michel Casevitz, "Remarques sur l'histoire de quelques mots exprimant l'espace en grec », Revue des Études Anciennes 100 (1998), p. 417-435.

17. Denis Rousset, Le territoire de Delphes et la terre d'Apollon (Bibliothèque des Écoles françaises d'Athènes et de Rome 310), Paris 2002, p. 194-195, no 29 , 19-26 (= CID IV, no 108; LSCG 79, daté de 178 av. n.è.), avec traduction, et p. 204. La délimitation des terres réservées aux vaches et aux juments s'étend « de la route qui mène à Astyron, en suivant la route menant à Paipalidas et jusqu'au Lakonikon, du Lakonikon vers la gauche jusqu'au vallon (napan) de Hierapeteia, [du vallon], en suivant la route de l'hippodrome, celle de l'étranger, qui va au L[- - - ] soit pour les vaches et les juments sacrées. [- - - ] mener les troupeaux des particuliers ». Pour la mise en culture des espaces sacrés loués, voir Carmine Ampolo, «I terreni sacri nel mondo greco », dans Elio Lo Cascio, Dominic W. Rathbone (éd.), Production and Public Powers in Classical Antiquity, Cambridge 2000, p. 14-19 (17). 
d'Éleusis, terre située à la frontière entre l'Attique et la Mégaride et dont les bornes sont contestées ${ }^{18}$. On s'adresse à l'oracle de Delphes afin de savoir s'il est «plus profitable et plus avantageux pour le peuple [athénien] que le basileus afferme les terrains maintenant cultivés [dans les limites de l'orgas sacrée], pour employer le revenu à la construction du portique et à l'entretien du sanctuaire des deux déesses ${ }^{19} »$. Si l'on suit cette lecture ${ }^{20}$, cette terre interdite à l'usage et à la culture ${ }^{21}$ a bien été cultivée par les Athéniens qui ont décidé de délimiter à nouveau le terrain ${ }^{22}$.

D'autres terres appartenant aux dieux sont mises en valeur et un règlement vient rappeler les obligations et les droits de celui qui en a la charge ${ }^{23}$. D'autres modalités d'utilisation montrent que les limites entre les propriétés des dieux et celles de la cité sont fluctuantes. En Chalcédoine au $\mathrm{I}^{\mathrm{er}}$ siècle avant notre ère ou $\mathrm{I}^{\mathrm{er}}$ siècle de notre ère, le règlement de la vente du sacerdoce d'Asclépios donne à l'acheteur l'usufruit des terres publiques qui entourent le sanctuaire, à moins que la cité ne veuille y construire ${ }^{24}$. La cité a donc priorité sur ses propres terres. Lorsqu'un sanctuaire est délimité, ce n'est d'ailleurs pas toujours pour sa protection. Les Béotiens ont accusé les Athéniens, lors de la campagne du Délion en 424,

18. Kevin Clinton, Eleusis. The Inscriptions on Stone. Documents of the Sanctuary of the Two Goddesses and Public Documents of the Deme, Athènes 2005, $\mathrm{n}^{\circ} 144$ (=IG II ${ }^{2} 204 ; L S C G$ 32). Voir James McDonald, «Athens and the hiera orgas », dans Matthew Dillon (éd.), Religion in the Ancient World: New Themes and Approaches, Amsterdam 1996, p. 321-332.

19. LSCG 32, 24-27.

20. Robert Parker, Miasma. Pollution and Purification in Early Greek Religion, Oxford 1996, p. 161, n. 99, considère qu'il faut plutôt lire ek]tos tôn horôn, supposant ainsi que les Athéniens veulent cultiver les terres immédiatement à l'extérieur des bornes, celles que Thucydide qualifie comme la gê aoristos par rapport à la gê hiera (I, 139, 2). Nous suivons ici la lecture de Kevin Clinton, Eleusis, II. Commentary, Athènes 2008, p. 139.

21. Voir notamment Thucydide, I, 139, 1; Pausanias, III, 4, 6. La réponse de l'oracle, négative, est rapportée par Philochore, FGrHist 328 F 155 et Androtion, FGrHist 324 F 30.

22. Un décret athénien daté d'environ 430 av. n.è., délimite de la même façon les sanctuaires du Pelargikon à l'intérieur duquel on ne peut fonder un autel, couper des pierres ou les emporter sans autorisation, voir $I G \mathrm{I}^{3} 78,54-58$ (= LSCG 5).

23. $I G$ IX 1, 654, 1-11 (= LSCG 86, Ithaque, $\mathrm{II}^{\mathrm{e}} \mathrm{s}$. de n.è.). Celui qui possède et exploite le terrain sacré à Artémis a l'obligation d'offrir une dîme chaque année et d'entretenir le temple. Cette inscription est identique à celle que cite Xénophon à propos de ses propres terres à Skillonte dédiées à Artémis (voir Anabase V, 3, 13). Il est donc possible qu'elle constitue un faux ou une copie tardive).

24. Sylß ${ }^{3} 1009,6$ (=LSAM 5). 
d'avoir fortifié le sanctuaire et de s'y être installés en y faisant tout ce qui se fait dans un lieu qu'on peut fouler (bebêlos $)^{25}$.

L'espace du sanctuaire correspond plus ou moins au temenos, l'espace découpé et assigné à une divinité ${ }^{26}$. Par rapport au terme hieron qui suggère la sacralité du lieu et qui est généralement traduit par «sanctuaire », le terme temenos, à travers la notion de découpage, met en lumière une autre caractéristique de l'espace sacré, le terrain réservé à un dieu ou à un héros ${ }^{27}$. Les deux termes en effet se recoupent sans nécessairement se superposer ${ }^{28}$. Ils apparaissent également côte à côte dans certaines inscriptions. Nous pouvons soit considérer qu'ils sont utilisés de façon interchangeable, soit que l'expression désigne l'ensemble des lieux apparte-

25. Thucydide, IV, 97,3 .

26. B. Bergquist, The Archaic Greek Temenos, op. cit., p. 5. Marie-Christine Hellmann, Recherches sur le vocabulaire de l'architecture grecque, d'après les inscriptions de Délos, École française d'Athènes-Paris 1992, p. 169, retient comme sens premier le « domaine appartenant à un héros ou à une divinité ». Selon Michel Casevitz, «Temples et sanctuaires : ce qu'apprend l'étude lexicologique », dans Georges Roux (dir.), Temples et sanctuaires. Séminaire de recherche 1981-1983 (Travaux de la Maison de l'Orient 7), 1984, p. 81-95 (85-86), chez Homère, temenos a le sens de domaine prélevé, réservé hors de l'ensemble des terres.

27. Vinciane Pirenne-Delforge, "Le lexique des lieux de culte dans la Périégèse de Pausanias ", Archiv für Religionsgeschichte 10 (2008), p. 143-178 (161). Notons que dans l'Iliade II, 506, le hieron de Poséidon à Onchestos est désigné comme un aglaon alsos. Selon M. Casevitz, "Temples et sanctuaires ", art. cité, p. 92, alsos, le bois sacré, équivaut dans ce cas à temenos. Voir aussi M. Horster, Landbesitz griechischer Heiligtümer, op. cit., p. 94 pour d'autres références. Pour l'alsos, voir Christian Jacob, « Paysage et bois sacré : alsos dans la Périégèse de la Grèce de Pausanias ", dans Les bois sacrés. Actes du Colloque International organisé par le Centre Jean Bérard et l'École Pratique des Hautes Études (Ve section), Naples, 23-25 novembre 1989 (Centre Jean Bérard 10), Naples 1993, p. 31-44. L'alsos est protégé comme le sont les autres espaces sacrés. À Chios, au IV ${ }^{\mathrm{e}} \mathrm{s}$., on interdit d'y faire paître les animaux ou d'y déposer du fumier $\left(S_{y l l}{ }^{3}\right.$ 986, 3-4 = LSCG 116). Sa relation au sanctuaire n'est toutefois pas toujours évidente. Dans la même inscription, après avoir précisé que le règlement doit être inscrit dans les bois (20-22: tauta grapsai en tois alsesin), on interdit d'emporter les objets sacrés hors du hieron (22-23). Il peut par ailleurs contenir diverses constructions $(I G$ IX 2, 583, 39 $=L S S 45$, Actium, après 217 av. n.è., règlement du culte d'Apollon). En somme, l'alsos semble recevoir la protection et bénéficier des mêmes interdits que d'autres lieux sacrés.

28. D'autres termes marquent aussi la délimitation du sanctuaire, comme peribolos. Par exemple, dans le peribolos de Zeus Olumpios à Athènes, Gè, la Terre qui porte l'épiclèse Olumpia possède un temenos, voir Pausanias, I, 18, 7. Pour d'autres exemples, voir M.-C. Hellmann, Recherches sur le vocabulaire, op. cit., p. 171. 
nant à la divinité dans un souci de complétude de l'énonciation ${ }^{29}$. L'expression to hieron kai to temenos se rencontre par exemple dans le règlement du culte d'Alektrônê à Ialysos, vers 300 avant notre ère ${ }^{30}$. L'interdiction d'apporter certains objets s'applique au temenos seul (10-12) alors que la loi (nomos) concerne to hieron kai to temenos (21). Dans un décret d'environ 160 de notre ère, relatif à la trêve à l'occasion d'une fête d'Artémis à Éphèse, la déesse apparaît si importante que " partout, des sanctuaires (hiera) et des domaines (temenê) lui sont consacrés, des temples (naoi) sont fondés $»^{31}$. Le hieron et le temenos peuvent servir de délimitation à l'intérieur de laquelle la coutume prescrit de ne pas introduire certains objets, comme au Letôon de Xanthos $^{32}$. D'après le règlement protégeant les arbres du sanctuaire d'Apollon à Koropé, vers 100 avant notre ère, les arbres qui se trouvent dans le sanctuaire (hieron) ont été détruits. Il est donc « nécessaire et utile que l'on montre quelque sollicitude à leur égard, de telle sorte que, par l'agrandissement, l'embellissement du temenos, la grandeur du lieu apparaisse de façon tout à fait manifeste ». À cette fin, on fait connaître à tous ceux qui viennent dans le sanctuaire (hieron) qu'il est interdit de couper les arbres "dans le lieu clairement indiqué » (en tôi diasaphoumenôi topôi $)^{33}$. Dans ce cas, le hieron semble englober le temenos.

La limite marquée par le temenos semble donc assez précise. C'est par exemple la limite au-delà de laquelle on ne peut emporter, lors de certains sacrifices, la viande ${ }^{34}$. On peut aussi interdire d'emporter les branches et les feuillages des arbres hors du hieron ${ }^{35}$. Toutefois,

29. C'est vraisemblablement le cas dans le décret concernant la restauration des sanctuaires, les hiera kai temenê attiques, voir $I G \mathrm{II}^{2} 1035,4$ (début du $\mathrm{I}^{\mathrm{er}} \mathrm{s}$. av. n.è.). Il est toutefois plus difficile de trancher lorsque des cas isolés mentionnent un hieron et un temenos sur les mêmes lieux.

30. IG XII 1, 677, 3 (= LSCG 136).

31. IK Ephesos 24, B 11-13 (= LSAM 31).

32. SEG 36 (1986), 1221; Christian Le Roy, «Un règlement religieux au Létôon de Xanthos », Revue Archéologique 1986, p. 279-300 (279-280), daté de la fin $\mathrm{du}_{\mathrm{III}}^{\mathrm{e}}-\mathrm{II}^{\mathrm{e}} \mathrm{s}$.

33. $I G$ IX $2,1109,4-8$ et $12(=L S C G 84)$.

34. $I G$ VII 235, 31-32 (= LSCG 69, Oropos, règlement du culte d'Amphiaraos, $\mathrm{IV}^{\mathrm{e}} \mathrm{s}$. av. n.è.). Pour l'interdiction d'emporter la viande, voir Gunnel Ekroth, The Sacrificial Rituals of Greek Hero-Cults in the Archaic to the early Hellenistic periods (Kernos Suppl. 12), Liège 2002, p. 313-325.

35. $I G \mathrm{II}^{2} 1362$, 5-7 (= LSCG 37, Attique, règlement protégeant les arbres du sanctuaire d'Apollon Erithaseos, fin du $\mathrm{IV}^{\mathrm{e}} \mathrm{s}$. av. n.è.). Pour la protection des 
pour ce qui est de la possession des terres, cette limite ne marque pas toujours une séparation bien nette entre l'intérieur et l'extérieur. À $\mathrm{Cos}$, le règlement $\mathrm{du} \mathrm{v}^{\mathrm{e}}$ siècle qui protège le bois d'Asclépios stipule que « si quelqu'un coupe les cyprès qui se trouvent à l'intérieur ou à l'extérieur du temenos ou s'il emporte le bois des cyprès coupés hors du temenos », il doit payer une amende. L'impiété est commise envers le sanctuaire dans l'ensemble (hieron). Cela signifie probablement que le sanctuaire possédait les terres environnantes du temenos. L'assemblée peut toutefois se servir de ce bois pour des œuvres publiques ${ }^{36}$. L'inscription qui lui succède porte la même interdiction qui ne concerne toutefois plus les terres environnantes : « afin que le temenos d'Apollon Kuparissios et d'Asclépios soit préservé, que personne ne coupe les cyprès à l'intérieur du lieu délimité par des bornes du temenos ${ }^{37} \gg$. Plus précis encore, un bail de Thespies daté d'environ 230 avant notre ère, permet au bailleur de cultiver les terres sacrées des Muses et stipule qu'il doit laisser une superficie de cent pieds autour du sanctuaire (hiaron) de Meilichios ${ }^{38}$.

Une fondation privée comme celle de Diomédon à Cos, vers 300 avant notre ère, comprend la consécration du temenos d'Héraclès Diomedonteios, de maisons pour les étrangers qui se trouvent dans le jardin et de bâtiments ${ }^{39}$. La consécration comprend donc des parties situées à l'extérieur du temenos. En effet, « il n'est permis à personne de s'approprier, de vendre ou d'hypothéquer ni les logements voisins du temenos ni le temenos lui-même ${ }^{40}$ ». Les statues et les offrandes se trouvent dans une oikia et doivent y rester ${ }^{41}$. De

espaces verts des sanctuaires, voir Eran Lupu, Greek Sacred Law. A Collection of New Documents (NGSL), Leyde-Boston 2005, p. 26-27.

36. $L S C G 150$, A 1-7 (fin du ve s.).

37. $L S C G 150$, B 1-8 (IV ou début du $\mathrm{III}^{\mathrm{e}}$ s. selon Susan M. Sherwin-White, Ancient Cos. An historical study from the Dorian settlement to the Imperial period, Göttingen 1978, p. 302, n. 99). L'interdiction de couper du bois est fréquente. Voir par exemple Andanie, $L S C G$ 65, 78 : «Que nul ne coupe (du bois) au lieu sacré (hierou topou)». Pour la protection des arbres en général, voir Matthew P.J. Dillon, «The Ecology of the Greek Sanctuary », Zeitschrift für Papyrologie und Epigraphik 118 (1997), p. 113-127 (115-116).

38. Michel Feyel, «Etudes d'épigraphie béotienne», Bulletin de Correspondance Hellénique 60 (1936), p. 175-183 (182), nº 2, 1. 27 (Henri W. Pleket, Epigraphica, I. Texts on the economic history of the Greek World, Leyde 1964, $\left.\mathrm{n}^{\circ} 45\right)$.

39. $L S C G 177$, A $1-4$.

40. Ibid., 43-47.

41. Ibid., B II 56-59. 
façon similaire, dans le cas de la phratrie des Klytidai de Chios qui réforment leur culte vers 335 avant notre ère, c'est dans un oikos temenios hieros, une maison sacrée dans le temenos, que sont apportés les objets sacrés ou les offrandes (hiera) provenant des maisons particulières ${ }^{42}$. Ces composantes, un temenos, une bâtisse et des hiera, sont en fait celles d'un sanctuaire.

Les règlements protégeant les sanctuaires énoncent les espaces à respecter avec précision. En Méonie, en 147/146, les restrictions cathartiques visent ceux qui entrent « dans le lieu circonscrit (periôrismenon topon) du Métroion ${ }^{43} »$. Les précisions des lieux à préserver s'accumulent parfois dans une sorte de progression dans l'espace, allant de l'entrée du sanctuaire vers le temple, suivant un parcours soumis aux mêmes interdictions. Une inscription de Lindos du $\mathrm{III}^{\mathrm{e}}$ siècle de notre ère précise les règles de pureté à suivre dans le sanctuaire de Pallas : "Il est conforme d'être pur et exempt de souillure pour entrer dans le lieu des perirrhantêria et des portes du temple (naos) ${ }^{44}$. » L'étranger pur peut s'avancer, et la fin de l'inscription stipule : «Si tu portes quelque possession (ou fardeau), quitte le temple (naos) sans le déposer, va où tu veux hors du sanctuaire (temenos) de Pallas ${ }^{45}$. »

Il y a pourtant des exemples qui suggèrent une gradation dans la sacralité de la terre. Cette gradation dans la sacralité de l'espace cultuel s'exprime aussi dans les règlements concernant la pureté requise pour l'accès aux sanctuaires ${ }^{46}$. Selon une inscription de Smyrne datée du II $^{\mathrm{e}}$-III ${ }^{\mathrm{e}}$ siècle de notre ère, il faut s'abstenir durant quarante jours d'entrer dans le temenos et les temples (naoi) de Bromios après l'exposition d'un nouveau-né ou un avortement. Mais il convient de se tenir durant un mois à l'écart du propulon, du vestibule du temple ou du sanctuaire, si la mort frappe un des

42. LSCG 118, 2-6. Sur le culte et sa réforme, voir Pierre Brulé, « La sainte maison commune des Klytides de Chios », Ktema 23 (1998), p. 307-324 (310-312).

43. $\operatorname{LSAM} 18,10-11$.

44. LSS 91, 1-3. Selon Lucien, Sur les sacrifices 13, une loi interdit «de s'avancer au-delà des perirrhantêria à celui qui n'a pas les mains pures ».

45. LSS 91, 25-26.

46. R. Parker, Miasma, op. cit., p. 162. Des étapes apparaissent à l'intérieur du temple lui-même, marquées par divers éléments architecturaux, voir Torsten Mattern, «Architektur und Ritual. Architektur als funktionaler Rahmen antiker Kultpraxis », dans Joannis Mylonopoulos, Hubert Roeder (éd.), Archäologie und Ritual. Auf der Suche nach der rituellen Handlung in den antiken Kulturen Ägyptens und Griechenlands, Vienne 2006, p. 167-183 (173-177). 
proches parents ${ }^{47}$. À Éresos, au II ${ }^{\mathrm{e}}$ siècle avant notre ère, il y a une différence entre ce que l'on peut introduire dans le temenos et ce que l'on peut introduire dans le naos. Les armes et les dépouilles d'animaux morts sont interdites au temenos. Pour le naos, les restrictions comprennent également les objets en fer et en bronze à l' exception des monnaies, ainsi que les vêtements en cuir ${ }^{48}$.

Nous avons vu que l'espace réservé du temenos est considéré dans son association au couple temple-autel comme un élément essentiel, un ensemble caractéristique et nécessaire des lieux de culte. C'est notamment l'espace dans lequel s'accomplissent le sacrifice et d'autres rites, à l'intérieur d'une ligne de démarcation réelle ou imaginaire $^{49}$. Il est censé séparer l'espace sacré de ce qui se trouve au-delà, du " profane ${ }^{50}$ ». Les périboles et autres vestiges matériels délimitant les sanctuaires vont dans ce sens. Outre la gradation dans la sacralité qui apparaît dans les inscriptions, la logique rituelle ellemême semble toutefois répondre à d'autres critères que la séparation du sacré-profane. Ainsi à Olympie, la procession qui passait devant les soixante-neuf ou soixante-dix autels pour sacrifier selon la manière ancienne, suit un parcours qui traverse le temenos de l'Altis à plusieurs reprises ${ }^{51}$. Selon les termes de Roland Étienne, "le cheminement de la procession n'est pas défini par un "dedans", le temenos proprement dit, et un "dehors", au-delà du péribole ${ }^{52} \gg$. Il apparaît dès lors clairement que les délimitations spatiales des lieux sacrés ne régissent pas le rite lui-même qui répond à une logique d'ordre différent.

La logique rituelle impose aussi de reconsidérer la distinction établie entre les éléments essentiels et les éléments non nécessaires à la constitution de l'espace du culte. Parmi les éléments considérés comme non essentiels, on trouve la stoa, l'oikos et les ensembles d'oikoi, les offrandes « votives », la végétation ${ }^{53}$, etc.

47. IK Smyrna 728, 2-7 (= LSAM 84).

48. IG XII 126, 12-17 (= LSCG 124).

49. John Pedley, Sanctuaries and the Sacred in the Ancient Greek World, Cambridge 2005, p. 6.

50. Ibid., p. 57. La même valeur symbolique est attribuée aux portes et aux propylées qui annonceraient ce passage du sacré au profane.

51. Pausanias, V, 14, 4-15, 11.

52. Roland Étienne, «Autels et sacrifices », dans Albert Schachter (éd.), Le sanctuaire grec (Fondation Hardt pour l'étude de l'antiquité classique, Entretiens 37, 1990), Genève 1992, p. 291-312 (306, n. 28 et fig. 2).

53. B. Bergquist, The Archaic Greek Temenos, op. cit., p. 6. 
Ce sont pourtant ces mêmes éléments qui caractérisent les grands sanctuaires ${ }^{54}$, et ils remplissent souvent des fonctions rituelles; le rite peut s'organiser autour d'un arbre ou s'accomplir à l'abri d'une stoa. Il faut dès lors revoir quelques structures à l'intérieur même du temenos désignées par des termes ambigus qui portent à confusion.

On a vu que le découpage du temenos ne semble pas correspondre nécessairement au hieron, qui désigne le sanctuaire dans le sens le plus large du terme ${ }^{55}$. Notons que le terme hieron est aussi ambigu que celui de « sanctuaire » lui-même ${ }^{56}$. D'un accord tacite, les chercheurs désignent comme un sanctuaire le lieu consacré à une divinité, quelles que soient les structures qu'il renferme, l'ensemble de ce qui se trouve à l'intérieur d'une enceinte, d'un peribolos ${ }^{57}$. Ce dernier terme n'a d'ailleurs pas de caractère à proprement parler religieux ${ }^{58}$ et il ne correspond pas nécessairement au temenos. Par exemple, dans une inscription d'Éphèse datée du $\mathrm{II}^{\mathrm{e}}$ siècle avant notre ère, " le temenos est tout entier un lieu inviolable, tout ce qui est à l'intérieur du péribole ${ }^{59} »$. Hieron peut par ailleurs désigner un temple et apparaître comme un synonyme de naos, terme qui désigne généralement une construction, un élément bâti, qu'il soit isolé ou partie d'un ensemble plus large ${ }^{60}$.

54. J. Pedley, Sanctuaries and the Sacred, op. cit., p. 6, sans parler d'éléments essentiels, reprend à peu près la même énumération que Bergquist (en ajoutant divers espaces et les offrandes) pour désigner les éléments qui, en quantité, taille, forme et matériau divers, se rencontrent dans les grands sanctuaires.

55. V. Pirenne-Delforge, « Le lexique des lieux de culte », art. cité, p. 145-151. Ce n'est pas le lieu de reprendre ici les divers emplois du terme qui caractérise aussi bien un lieu, un objet etc. Pour une synthèse, voir Jean Rudhardt, Notions fondamentales de la pensée religieuse et actes constitutifs du culte dans la Grèce classique, Paris 1992 [1958], p. 22-30 et Jean Casabona, Recherches sur le vocabulaire des sacrifices en grec, des origines à la fin de l'époque classique, Aix-enProvence 1966, p. 5-18.

56. « Sanctuaire » désigne en français le « lieu le plus saint d'un édifice religieux », un « lieu saint » ou un «édifice consacré à la pratique d'un culte», voir Trésor de la langue française informatisé, s.v.

57. Comme le remarque J. Rudhardt, «La perception grecque du territoire sacré », art. cité, p. 176, n. 3, le sanctuaire désigne un territoire, un lieu sacré, sans qu'il soit marqué par quelque élément caractéristique.

58. V. Pirenne-Delforge, " Le lexique des lieux de culte », art. cité, p. 167.

59. IK Ephesos 1520, 1-2 (= LSAM 85).

60. M. Casevitz, «Temples et sanctuaires », art. cité, p. 87; V. PirenneDelforge, "Le lexique des lieux de culte», art. cité, p. 147-148. Pour hieron comme bâtiment, voir M.-C. Hellmann, Recherches sur le vocabulaire, op. cit., p. 170. La même ambiguïté apparaît avec le terme sêkos, à la fois enceinte, en 
Or naos peut également désigner du mobilier, en l'occurrence un «temple» portatif ${ }^{61}$. Il nous faut donc décrire plus précisément cette construction, en tenant compte des différentes formes de temples en relation avec les dispositifs rituels qu'ils renferment ou qui les entourent.

\section{UN BÂTIMENT POUR LE CULTE}

Le terme naos peut désigner la construction du temple dans son ensemble ou bien il peut s'opposer au pronaos, la partie antérieure du bâtiment, et désigner ainsi ce qu'on appelle, comme le faisait d'ailleurs Vitruve, la cella, la partie centrale du bâtiment.

De façon significative, le temple, qui est pour nous « le monument caractéristique des sanctuaires ${ }^{62} »$, n'est pas un élément indispensable aux lieux de culte. Il peut être absent, ou bien plusieurs temples d'une même divinité peuvent coexister dans un sanctuaire ${ }^{63}$. La question se pose alors de savoir si ces bâtiments avaient tous la même fonction cultuelle.

De la même façon que naos et hieron peuvent apparaître comme interchangeables, naos et thêsauros peuvent parfois être utilisés indifféremment. De la même manière aussi, thêsauros peut désigner une pièce de mobilier. Dans les inscriptions déliennes, il désigne de façon constante les troncs à offrandes. Le trésor, le bâtiment en forme de naos dédié par une cité pour renfermer les offrandes précieuses, y est désigné par le terme oikos. Du point de vue étymologique, l'oikos désigne une habitation et le terme ne peut recevoir un

contexte religieux ou pas, et partie reculée du temple (cella). Voir M. Casevitz, « Temples et sanctuaires », art. cité, p. 94 et M.-C. Hellmann, Recherches sur le vocabulaire, op. cit., p. 368.

61. Hérodote, II, 63, décrit ainsi un naos à Paprémis en Égypte. Tandis que les prêtres se tiennent à l'entrée du sanctuaire, du hiron, « la statue, contenue dans un petit nêos en bois doré, a été transportée la veille dans un autre édifice sacré (oikêma hiron) ». Jusque-là nous pourrions comprendre que la statue a été transportée dans un autre temple. La suite de la description fait toutefois état d'un naos portatif: " les quelques [prêtres] laissés autour d'elle traînent un char à quatre roues qui porte le nêos et la statue que le nêos contient».

62. Georges Roux, "Trésors, temples, tholos », dans Temples et sanctuaires, op. cit., p. 153-171 (153).

63. Ainsi Apollon en compte deux dans son sanctuaire de Délos au début de la période de l'indépendance. 
sens plus précis que dans un contexte particulier ${ }^{64}$. Il arrive également que oikos ait le sens d'habitation des dieux ${ }^{65}$.

Pausanias désigne par le terme naoi les thêsauroi du sanctuaire (hieron) d'Athéna Pronaia à Delphes. De son temps, le premier était en ruines, le second était vide de statues de dieux et d'hommes, le troisième contenait des statues d'empereurs romains. Le quatrième est celui « de Pronaia $^{66} »$. Quelles que soient les identifications de ces naoi sur le site ${ }^{67}$, Pausanias les désigne tous du même nom, sans préciser davantage leur fonction. De même, Polémon appelle naoi les trésors de Métaponte et de Byzance à Olympie ${ }^{68}$, tout comme Diodore de Sicile définit le trésor fondé par les Thébains à Delphes comme le naos des Phocidiens ${ }^{69}$.

Georges Roux posait ainsi la question de savoir « si les Grecs établissaient entre ces deux catégories d'édifices une distinction aussi nette que nous le faisons $\rangle^{70}$. Il remarquait à juste titre que le naos grec, que nous traduisons invariablement par temple ou par cella, n'est pas toujours un lieu de culte. L'exemple du Parthénon est explicite à cet égard. Il abrite la statue chryséléphantine d'Athéna faite par Phidias. Dans l'opisthodome se trouvent les biens, les trésors d'Athéna et des autres dieux; en somme, le Parthénon luimême apparaît comme un trésor monumental. De la même manière, le «temple (neôs) des Athéniens » ou «temple aux sept statues»

64. M.-C. Hellmann, Recherches sur le vocabulaire, op. cit., p. 156. Oikos alterne dans les inscriptions de Delphes avec thêsauros pour désigner le trésor des Athéniens, voir Marie-Christine Hellmann, «Caractères de l'épigraphie architecturale à Delphes ", dans Delphes cent ans après la Grande fouille. Essai de bilan. Actes du colloque organisé par l'EFA, 17-20 septembre 1992 (Bulletin de Correspondance Hellénique Suppl. 36), Athènes-Paris 2000, p. 167-177 (176).

65. Hérodote, VIII, 143. Voir aussi l'oikos temenios hieros des Klytidai de Chios ( $L S C G 118,3)$.

66. Pausanias, X, 8, 6. Selon G. Roux, "Trésors, temples, tholos », art. cité, p. 158 , la description est si sommaire qu'il s'agit peut-être simplement d'une négligence de style.

67. Voir notamment Christian Le Roy, « Pausanias à Marmaria », dans Études delphiques (Bulletin de Correspondance Hellénique Suppl. 4), Athènes-Paris 1977, p. 247-271, qui passe en revue les diverses identifications.

68. Athénée, XI 479 F-480A. Polémon est l'auteur d'un traité sur les thêsauroi de Delphes, voir Plutarque, Propos de table, V, 2 (675 B).

69. Diodore de Sicile, XVII, 10, 5.

70. G. Roux, «Trésors, temples, tholos», art. cité, p. 159; idem, «Le vrai temple d'Apollon à Délos », Bulletin de Correspondance Hellénique 111 (1979), p. 119-135 (110-111), où il propose l'appellation générique de «templestrésors ». 
à Délos, abritait les statues que les Athéniens avaient offertes à Apollon après la paix de Nicias en $421^{71}$. Plus déroutante est la description que donne Pausanias de l'Érechthéion. Véritable lieu de culte, il est désigné comme un oikêma double ${ }^{72}$ contenant divers autels, celui de Poséidon sur lequel les Athéniens sacrifient aussi à Érechthée, celui de Boutès et celui d'Héphaïstos.

Ces quelques occurrences montrent que les bâtiments que nous désignons comme des temples, présupposant de la sorte des lieux de culte, devraient être qualifiés non pas d'après leur forme ${ }^{73}$ mais $d$ 'après leur fonction qui, elle aussi, peut varier dans le temps. Ainsi le temple d'Héra à Olympie, véritable lieu de culte de la déesse, a été probablement par la suite, en tous les cas à l'époque de Pausanias, converti en trésor ${ }^{74}$.

Le temple apparaît parmi les éléments essentiels du sanctuaire, notamment dans son association avec l'autel. Les diverses dénominations d'autels ont été longuement étudiées et dépassent le cadre de cette analyse. Considéré dans sa relation avec le temple, dans son axe, l'autel assurerait par sa position la visibilité du rite sacrificiel à la fois pour les spectateurs humains et pour la divinité honorée $^{75}$. Cela implique que cette dernière réside dans son temple. Outre le fait que l'autel puisse se situer à l'intérieur du temple luimême $^{76}$, seule la fonction du bâtiment - et l'on vient de voir qu'elle doit dans la majorité des cas être clairement déterminée - permet d'avancer l'hypothèse d'un temple résidence divine. Il arrive toutefois dans le langage archéologique, lorsqu'on déduit la fonction de la forme, qu'on parle de recinto, d'enclos, à propos d'un bâtiment qui comporte un autel, ou d'un oikos à propos d'un bâtiment

71. G. Roux, « Trésors, temples, tholos », art. cité, p. 160.

72. Pausanias, I, 26, 5.

73. V. Pirenne-Delforge, « Le lexique des lieux de culte », art. cité, p. 152.

74. Karim W. Arafat, "Pausanias and the temple of Hera at Olympia », The Annual of the British School at Athens 90, 1995, p. 461-473 (470). Voir les statues et les objets que mentionne Pausanias, V, 17-20, 3, dans l'Héraion.

75. J. Pedley, Sanctuaries and the Sacred, op. cit., p. 8 et 60.

76. Georges Roux, «L'autel dans le temple », dans Roland Étienne, MarieThérèse Le Dinahet (éd.), L'espace sacrificiel dans les civilisations méditerranéennes de l'antiquité. Actes du Colloque tenu à la Maison de l'Orient, Lyon, 4-7 juin 1988, Paris 1991, p. 297-302, à propos de Samothrace, peut-être aussi Éleusis, et d'autres édifices, comme le Keratôn de Délos, spécialement construit pour abriter l'autel de cornes d'Apollon. 
qui a vraisemblablement servi au culte ${ }^{77}$. En utilisant des termes qui ne font pas spécifiquement référence au domaine religieux, on minimise la fonction cultuelle de ces structures. On applique alors le raisonnement qui faisait identifier un naos et un thêsauros par les anciens ${ }^{78}$. Roland Étienne doutait depuis longtemps que « le couple temple-autel soit un élément primordial - au sens fort - dans l'aménagement d'un sanctuaire ${ }^{79} »$, mettant en revanche en valeur les éléments «secondaires », qui prennent leur sens plein au point de vue rituel et définissent ainsi l'espace sacré. C'est effectivement au point de vue rituel que l'hypothèse d'éléments essentiels, le temenos avec un temple et un autel, pose problème; ce sont là en fait des éléments monumentaux auxquels il n'est que trop facile de s'attacher ${ }^{80}$. Seules les traces du rite permettent de reconstruire un espace qui, loin d'être désordonné ou accidentel, est structuré par des dispositifs aussi nombreux que variés. Il faut pour cela comprendre la fonction de chaque structure, cesser d'en considérer certaines comme cultuelles par inertie et inclure d'autres qui, pour ne pas être monumentales, sont souvent délaissées. Elles sont en effet destinées, ou participent à des degrés variables, à l'accomplissement du rite et, dans le cadre du sanctuaire, au culte qui honore la divinité maîtresse des lieux.

77. S'agissant respectivement du «Santuario ctonio » d'Agrigente - malgré le titre de l'article de Domenico Pancucci, «I Temenoi del santuario delle divinità ctonie ad Agrigento », dans Maria José Fontana, Maria Teresa Piraino et Francesco Paolo Rizzo (éd.), Miscellanea di studi classici in onore di Eugenio Manni 5, Rome 1980, p. 1665-1676, l'usage de parler de recinti prédomine - et du sanctuaire de Déméter et Corè sur l'Acrocorinthe, voir Nancy Bookidis, Ronald S. Stroud, The Sanctuary of Demeter and Kore: topography and architecture (Corinth 18, 3), Cambridge 1997, p. 64-73.

78. Sur la confusion de la configuration avec la fonction, voir Philippe Bruneau, «Sources textuelles et vestiges matériels : réflexions sur l'interprétation archéologique », dans Mélanges helléniques offerts à Georges Daux, Paris 1974, p. 33-42 (39). Voir aussi G. Roux, « Trésors, temples, tholos », art. cité, p. 163, qui associait les plans originaux des temples aux impératifs religieux, bien que nous ne puissions pas le suivre jusqu'au bout lorsqu'il considère que cet impératif d'ordre topographique concerne le respect du sol sacré recouvert par le temple.

79. R. Étienne, «Autels et sacrifices », art. cité, p. 305.

80. O. de Cazanove, J. Scheid, « Aux sources d'un colloque », art. cité, p. 3. 


\section{ABRÉVIATIONS}

CID IV : François Lefèvre et al., Documents amphictioniques. Corpus des inscriptions de Delphes, IV, École française d'Athènes-Paris 2002.

FGrHist : Felix Jacoby (éd.), Die Fragmente der griechischen Historiker, Berlin-Leyde 1923-1958.

IK Ephesos : Hermann Wankel et al. (éd.), Die Inschriften von Ephesos (Inschriften griechischer Städte aus Kleinasien XI, 1), Bonn 1979, et XV, 5, Bonn 1980.

IK Smyrna: Georg Petzl (éd.), Die Inschriften von Smyrna (Inschriften griechischer Städte aus Kleinasien XXIII-XXIV, II. 1), Bonn 1987.

LSAM : Franciszek Sokolowski, Les lois sacrées de l'Asie Mineure, Paris 1955.

LSS : Franciszek Sokolowski, Les lois sacrées des cités grecques. Supplément, Paris 1962.

LSCG : Franciszek Sokolowski, Les lois sacrées des cités grecques, Paris 1969.

SEG : Supplementum Epigraphicum Graecum, Leyde 1923-.

Syll $l^{3}$ : Wilhelm Dittenberger, Sylloge Inscriptionum Graecarum, Leipzig 1920.

ioanna.patera@gmail.com

ANHIMA

2, rue Vivienne

75002 Paris 